\title{
Immunosuppression induced by apoptosis of mixed lymphocyte culture is associated with p53
}

\author{
FULING WEI $^{1}$, YONGMEI CAO ${ }^{2}$, MENGRAN ZHANG $^{3}$, RUI HUANG ${ }^{4}$, \\ JUN CHENG ${ }^{3}$, GUOAN XIANG ${ }^{4}$ and JINQIAN ZHANG ${ }^{3}$ \\ ${ }^{1}$ Department of Cardiovascular Surgery, The PLA 309 Hospital, Beijing 100091; \\ ${ }^{2}$ International Mongolian Hospital, Hohhot of Inner Mongolia, Hohhot 010065; ${ }^{3}$ Institute of Infectious Diseases, \\ Beijing Ditan Hospital, Capital Medical University, Beijing 100015; ${ }^{4}$ Department of General Surgery, \\ The Second People's Hospital of Guangdong Province, Guangzhou 510515, P.R. China
}

Received October 14, 2012; Accepted January 15, 2013

DOI: $10.3892 / \mathrm{mmr} .2013 .1292$

\begin{abstract}
A lymphocyte inhibition model was created using a co-culture of donor and host lymphocytes, resulting in apoptosis of the latter, and subsequently, inducing immune tolerance. This method may be used to resolve the immune rejection problem prior to organ transplantation. Using mixed lymphocyte culture (MLC) and/or addition of anti-anti-IL-2 neutralizing monoclonal antibody, we successfully developed a lymphocyte inhibition model in vitro. In this model, the apoptosis of recipient lymphocytes co-cultured with donor lymphocytes was observed by Wright-Giemsa stain, electron microscopy imaging and flow cytometry. The growth and proliferation of mixed lymphocytes were detected by XTT and BrdU assays. Cell viability was determined using CCK-8 cell viability assay. The activity of the recipient lymphocytes was very high when stimulated by antigens alone [PMLC + D2 (Bm) group] but markedly lowered by anti-anti-IL-2 neutralizing monoclonal antibody [PMLC + D2 (Bm) + anti-IL-2 group]. The suppression of recipient lymphocyte activity was due to apoptosis mediated by p53 and caspase-3, and the optimal ratio of donor and recipient lymphocytes for apoptosis was explored. With the exception of the control group, the ratio of apoptotic cells was highest in the PMLC + D2 (Bm) + anti-IL-2 group and lowest in the PMLC + D2 (Bm) group. Blockade of IL-2 with anti-IL-2 neutralizing antibody resulted in an increased number of apoptotic lymphocytes in our experiment, which suggested that IL-2 inhibits the apoptosis of lymphocytes. These data suggest that IL-2 is involved in MLC-induced apoptosis of recipient lymphocytes, and that apoptosis may be associated with p53 and caspase- 3 pathways.
\end{abstract}

Correspondence to: Dr Jinqian Zhang, Institute of Infectious Diseases, Beijing Ditan Hospital, Capital Medical University, 8 Jingshun East St., Beijing 100015, P.R. China

E-mail: jingwanghou@yahoo.com.cn

Key words: immunosuppression, apoptosis, mixed lymphocyte culture, p53

\section{Introduction}

Organ transplantation is one of the greatest advances in modern medical science, but immune rejection following organ transplantation may cause tissue damage and even fatality. Transplant rejection occurs when the lymphocytes of the recipient recognize the allogenic antigens carried by the donor organ, and the recipient's immune system starts a process of attacking the transplanted organ. In the majority of cases, the transplant rejection response is moderate to severe, and it is almost inevitable in the allograft. Therefore, researchers are interested in studying immune suppression following organ transplantation and induction of immune tolerance prior to organ transplantation.

There are two possible mechanisms of allorecognition initiated by host $\mathrm{T}$ lymphocytes: the direct method (in acute rejection) and the indirect method (in chronic rejection). IL-2, a T-lymphocyte growth factor, plays an important role in $\mathrm{T}$ lymphocyte-mediated transplant rejection. The acute rejection is associated with elevated IL-2 levels in cyclosporine-treated patients. A significant change in IL-2 mRNA level in transplanted kidney could be detected 2 days prior to rejection (1-3). Rimm et al (4) demonstrated that the IL-2 mRNA level of the graft was correlated with acute rejection. Moderate rejection, on the contrary, was associated with decreased IL-2 production. The fixed TCR- $\beta$ chain caused a defective response to alloantigen, which was measured as decreased IL-2 generation and utilization, followed by an abnormally decreased graft-versus-host response (GVHR). Another opposite opinion, however, deemed that IL-2 induced immune tolerance via regulatory $\mathrm{T}$ cells $(5-8)$.

Immune tolerance related to organ transplantation is defined as a characterized state induced by the absence of a specific, deleterious immunological reaction against an allograft. It can be maintained with immunosuppressant drugs. Certain researchers (8) observed that donor antigens were capable of inducing death of lymphocytes in certain conditions. Transfusion of the donor antigen to the thymus of the recipient prior to organ transplantation induces immune tolerance in animals (9-11) and donor-specific transfusion prior to organ transplantation alleviates immune rejection in clinical 
trials $(12,13)$. Therefore, it is reasonable to propose that donor antigen could inhibit the lymphocytes of the recipient and even induce their death.

Due to the complicated internal environment, the observed suppressive effect in vivo is different from that in vitro, and is also unstable. To resolve the immune rejection problem ahead of organ transplantation, a lymphocyte inhibition model was established by co-culture of recipient and donor lymphocytes in order to induce immune tolerance. We co-cultured lymphocytes from different individuals in vitro, who could be regarded as donor and recipient, respectively, and detected the activities of lymphocytes in order to explore the mechanism of the activity change.

\section{Materials and methods}

Ethics. The study was approved by the Ethics Committee of Department of Cardiovascular Surgery, the PLA 309 Hospital, Beijing, China according to the Declaration of Helsinki. Written informed consent was obtained from the patients.

\section{Cell preparation}

Preparation of receptor cells. Blood (from a healthy control) was added to lymphocyte isolation liquid in a centrifuge tube, centrifuged at $1,000 \mathrm{rpm}$ for $20 \mathrm{~min}$, the mist-like buffy coat in the middle of the tube was imbibed, washed with RPMI-1640 medium and stained with trypan blue. More than $95 \%$ of the cells were alive, and then the live cells were counted. The cell concentration was diluted to $1 \times 10^{7}$ cells $/ \mathrm{ml}$ with RPMI-1640 containing $20 \%$ fetal bovine serum. The suspension was noted as $\mathrm{R}$.

Preparation of donor (D) lymphocytes. Another sample of blood (from a healthy control) was added to lymphocyte isolation liquid in a centrifuge tube, centrifuged at 1,000 rpm for $20 \mathrm{~min}$, the mist-like buffy coat in the middle of the tube was imbibed, washed with NS liquid and then the number of cells was counted. The suspension of the lymphocytes of the donor, after being inactivated with mitomycin, was noted as $\mathrm{D}(\mathrm{Bm})$. Half of D (Bm), noted as D1 (Bm), was diluted to $1 \times 10^{7}$ cells/ml with RPMI-1640 medium containing $20 \%$ fetal bovine serum. The other half of $\mathrm{D}(\mathrm{Bm})$, noted as D2 (Bm), was divided into 10 aliquots, and stored at $-70^{\circ} \mathrm{C}$.

Mixed lymphocyte culture (MLC). Lymphocyte specimens $\mathrm{R}$ and D1 (Bm) were mixed into 6-well plates (10 wells in total), cultured at $37^{\circ} \mathrm{C}$ in a $5 \% \mathrm{CO}_{2}$ incubator for $72 \mathrm{~h}$, and this was noted as the MLC. On the fourth day, MLC in the first well was collected, washed with RPMI-1640 medium and then counted. The cell concentration was diluted to $1 \times 10^{6}$ cells $/ \mathrm{ml}$ with RPMI-1640 containing 20\% fetal bovine serum, and the suspension was noted as PMLC (primed mixed lymphocyte culture) (14). An aliquot of frozen D2 (Bm) was washed with RPMI-1640 medium and then counted. The cell concentration was diluted to $1 \times 10^{6} / \mathrm{ml}$ with RPMI-1640 containing $20 \%$ fetal bovine serum.

RPMI-1640 liquid (100 $\mu \mathrm{l})$ was used as a blank control, and $50 \mu 1$ PMLC was used as a control. PMLC and D2 (Bm) were grouped in the following ways: i) PMLC $50 \mu \mathrm{l}$ and D2 (Bm) $50 \mu \mathrm{l}$; ii) PMLC $50 \mu \mathrm{l}$ and IL-2 purified neutralizing monoclonal antibody $15 \mu \mathrm{l}$; iii) PMLC $50 \mu \mathrm{l}$, D2 (Bm) $50 \mu \mathrm{l}$ and IL-2 purified neutralizing monoclonal antibody $15 \mu \mathrm{l}$. The mixtures were respectively divided into 96 -well plates and cultured in an incubator at $37^{\circ} \mathrm{C}$ in $5 \% \mathrm{CO}_{2}$ for $24 \mathrm{~h}$.

IL-2 monoclonal neutralizing antibody was provided by Professor Boquan Jin (The Fourth Military Medical University, Xi'an, China).

Wright-Giemsa stain. Following culture and collection, the cells were diluted to a concentration of $1 \times 10^{6}$ cells $/ \mathrm{ml}$. The cells were then smeared, fixed with $4 \%$ formaldehyde, stained with Wright-Giemsa for 3-4 min and then washed with distilled water until the edges showed a faint pinkish red. The smear was made transparent with xylene, and finally cell morphology was observed by microscopy.

Electron microscope detection. In order to obtain transmission electron microscope (TEM) images, cells were harvested from flasks with a cell scraper and washed twice with PBS prior to fixing with $5 \%$ glutaraldehyde for $1 \mathrm{~h}$ at $4^{\circ} \mathrm{C}$. The pellet was fixed with $1 \%$ osmium tetroxide $\left(\mathrm{OsO}_{4}\right)$ and dehydrated in a graded series of cold ethanol $(30,50,70,95$ and $100 \%$ ethanol, respectively). Finally, pellets were embedded in $100 \%$ pure resin (Durcupan ACM, Fluka, Buchs, Switzerland) (15). Thick $(1.5 \mu \mathrm{m})$ and ultrathin sections $(\sim 50 \mathrm{~nm})$ were cut with a Sorvall Porter-Blum MT2-B ultramicrotome (Porter, USA). Ultrathin sections were collected on copper grids and stained with uranyl acetate (5\% uranyl acetate in $70 \%$ ethanol) and $0.3 \%$ lead citrate. Unstained ultrathin sections were observed with a Philips CM200 TEM to identify the condition of the lymphocyte membrane, nucleus, chromatin, nuclear envelope and apoptotic body.

Cell growth and proliferation assay. Cell growth was determined by the colorimetric tetrazolium-derived XTT (sodium 3'-[1-(phenylaminocarbonyl)-3,4-tetrazolium]-bis(4-methoxy-6nitro) benzene sulfonic acid hydrate) assay (Roche Applied Science, Mannheim, Germany) and DNA synthesis of cells was assessed by the bromodeoxyuridine (BrdU) incorporation assay (Roche Applied Science). For the cell growth and proliferation assay, at $48 \mathrm{~h}$ after transfection the cells of each group were re-seeded in 96-well plates at a density of $0.3-1 \times 10^{4}$ cells/well. After $48 \mathrm{~h}$, XTT and incorporated BrdU were measured colorimetrically using a microtiter plate reader (Bio-Rad, Hercules, CA, USA) at a wavelength of $450 \mathrm{~nm}(16)$.

Cell viability assay. Cell viability was determined using a CCK-8 cell viability assay kit (Dojindo Laboratories, Kumamoto, Japan). All cells $\left(5 \times 10^{3}\right.$ cells/well) were pretreated according to the manufacturer's instructions and then incubated with or without $0.1 \mathrm{mM} \mathrm{H}_{2} \mathrm{O}_{2}$ for $16 \mathrm{~h}$ in a 96 -well plate. Cell viability assay kit solution $(10 \mu \mathrm{l})$ was added to each well. Following incubation for $1 \mathrm{~h}$ at $37^{\circ} \mathrm{C}$ in the dark, absorbencies were measured at $450 \mathrm{~nm}$ using a multiwell plate reader (17).

Determination of apoptosis. Apoptotic cells were identified by fluorescence-activated cell sorting (FACS) using Annexin V-Fluos (Biolegend, San Diego, CA, USA) according to the manufacturer's instructions. Apoptosis was verified by detection of activated caspases and p53. 


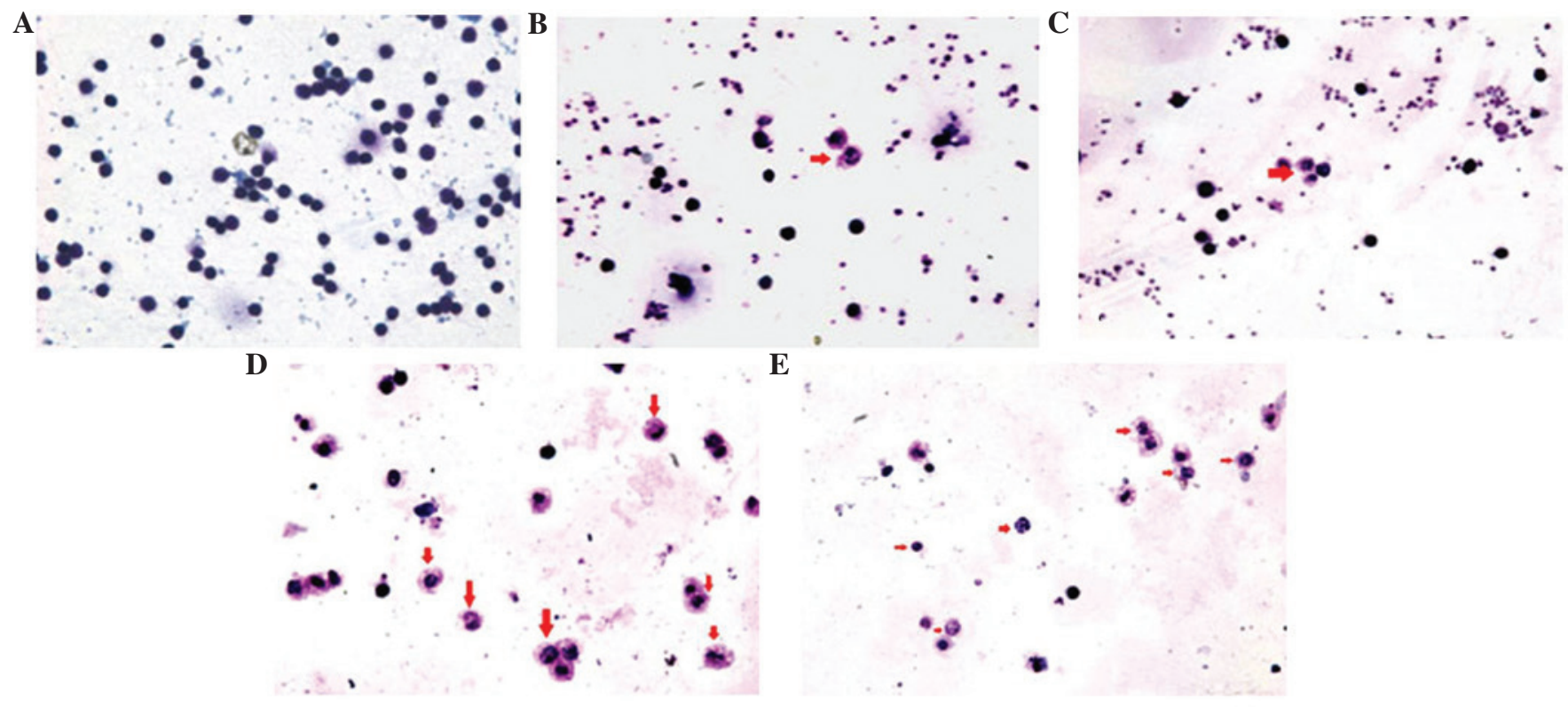

Figure 1. Lymphocyte staining with Wright-Giemsa at $24 \mathrm{~h}$. The apoptotic cells exhibited deep blue karyotin staining, nuclear atypia, chromatin margination, apoptotic bodies, karyopyknosis and schizolysis, but not so the cells in (A) the normal group. The counts of apoptotic cells in the (B) PMLC and (C) PMLC + D2 (Bm) groups were significantly lower than those in the (D) PMLC+anti-IL-2 and (E) PMLC + D2 (Bm) + anti-IL-2 groups. (E) In the PMLC + D2 (Bm) + anti-IL-2 group, the number of apoptotic lymphocytes was much lower than in the (D) PMLC+anti-IL-2 group. The arrows indicate the apoptotic cells (x400).

Antibodiesandimmunoblotting.Immunoblotting was performed as described previously (14). The following antibodies were used for immunoblotting at the indicated dilutions: p53 antibody (Santa Cruz Biotechnology, Inc., Santa Cruz, CA, USA; 1:1,000), caspase-3 (Cell Signaling Technology; 1:1,000) and $\beta$-actin (Santa Cruz Biotechnology, Inc.; 1:1,000). All affinitypurified and species-specific fluorophore-conjugated secondary antibodies were obtained from Santa Cruz Biotechnology, Inc. and used at dilutions between 1:500 and 1:800.

Statistics. All data are shown in the form of the means \pm SD. Significance was determined using the paired Student's t-test and one-way ANOVA test on the mean values of 3 different experiments. SNK-q was used to compare between 2 groups. All data were analyzed with SPSS 10.0 software for Windows $^{\mathrm{TM}}$. $\mathrm{P}<0.05$ was considered to indicate a statistically significant difference.

\section{Results}

Lymphocytes stained with Wright-Giemsa. The lymphocytes of each group were stained with Wright-Giemsa at an appropriate time point. All apoptotic cells exhibited deep blue karyotin staining, nuclear atypia, chromatin margination, apoptotic bodies, karyopyknosis and nuclear schizolysis, but the normal group did not (Fig. 1A). The counts of apoptotic cells in the PMLC (Fig. 1B) and PMLC + D2 (Bm) groups (Fig. 1C) were markedly lower than those in the PMLC + anti-IL-2 (Fig. 1D) and PMLC + D2 (Bm) + antiIL-2 groups (Fig. 1E). In the PMLC + D2 (Bm) + anti-IL-2 group (Fig. 1E), the counts of lymphocytes were lower than those in the PMLC + anti-IL-2 group (Fig. 1D).

Apoptosis of lymphocytes observed by TEM. The lymphocytes were observed by TEM (x10,000). Nuclear atypia, chromatin margination, karyopyknosis and schizolysis, as well as disappearance of nuclear envelopes and integration of cellular membrane, was observed in the images, particularly in the PMLC + anti-IL-2 group.

Blockade of IL-2 with anti-IL-2 purified neutralizing monoclonal antibody inhibits lymphocytes growth and proliferation. The lymphocyte growth in the PMLC + D2 (Bm) + anti-IL-2 group was lower than that in the PMLC and PMLC + D2 $(\mathrm{Bm})$ group $(\mathrm{P}<0.01)$ as detected by XTT assay, but not in the PMLC + anti-IL-2 group (Fig. 2A). The results of lymphocyte DNA synthesis analyzed with BrdU showed that the DNA change in the PMLC + D2 $(\mathrm{Bm})+$ anti-IL-2 group was less than that in the PMLC and PMLC + D2 $(\mathrm{Bm})$ group $(\mathrm{P}<0.01)$, but not in the PMLC + anti-IL-2 group (Fig. 2B). Cell viability was evaluated by CCK-8 assay (Fig. 2C) and lymphocyte proliferation was evaluated by cell count (Fig. 2D). The results were similar to those from the XTT assays. The proliferation of lymphocytes in the PMLC + anti-IL-2 group $(\mathrm{P}<0.01)$ was significantly inhibited, but not in the PMLC + D2 (Bm) and PMLC + anti-IL-2 groups ( $\mathrm{P}>0.05)$.

Blockade of IL-2 with anti-IL-2 purified neutralizing monoclonal antibody promotes lymphocyte apoptosis. The effects of MLC, as well as IL-2 purified neutralizing monoclonal antibody, on apoptosis distribution were determined by flow cytometry. The counts of apoptotic lymphocytes in the PMLC + anti-IL-2 group $(11.25 \pm 0.78 \%)$ and the PMLC + D2 $(\mathrm{Bm})+$ anti-IL-2 group $(31.65 \pm 1.33 \%)$ were much higher than in the PMLC $(5.95 \pm 0.24 \%)$ or PMLC + D2 (Bm) groups $(2.15 \pm 0.04 \%)$, respectively $(\mathrm{P}<0.01)$. The difference between the PMLC + anti-IL-2 and PMLC + D2 (Bm) + anti-IL-2 groups was also statistically significant $(\mathrm{P}<0.01$; Fig. 3$)$. The results revealed that MLC and IL-2 purified neutralizing monoclonal antibody significantly induced apoptosis of lymphocytes. 

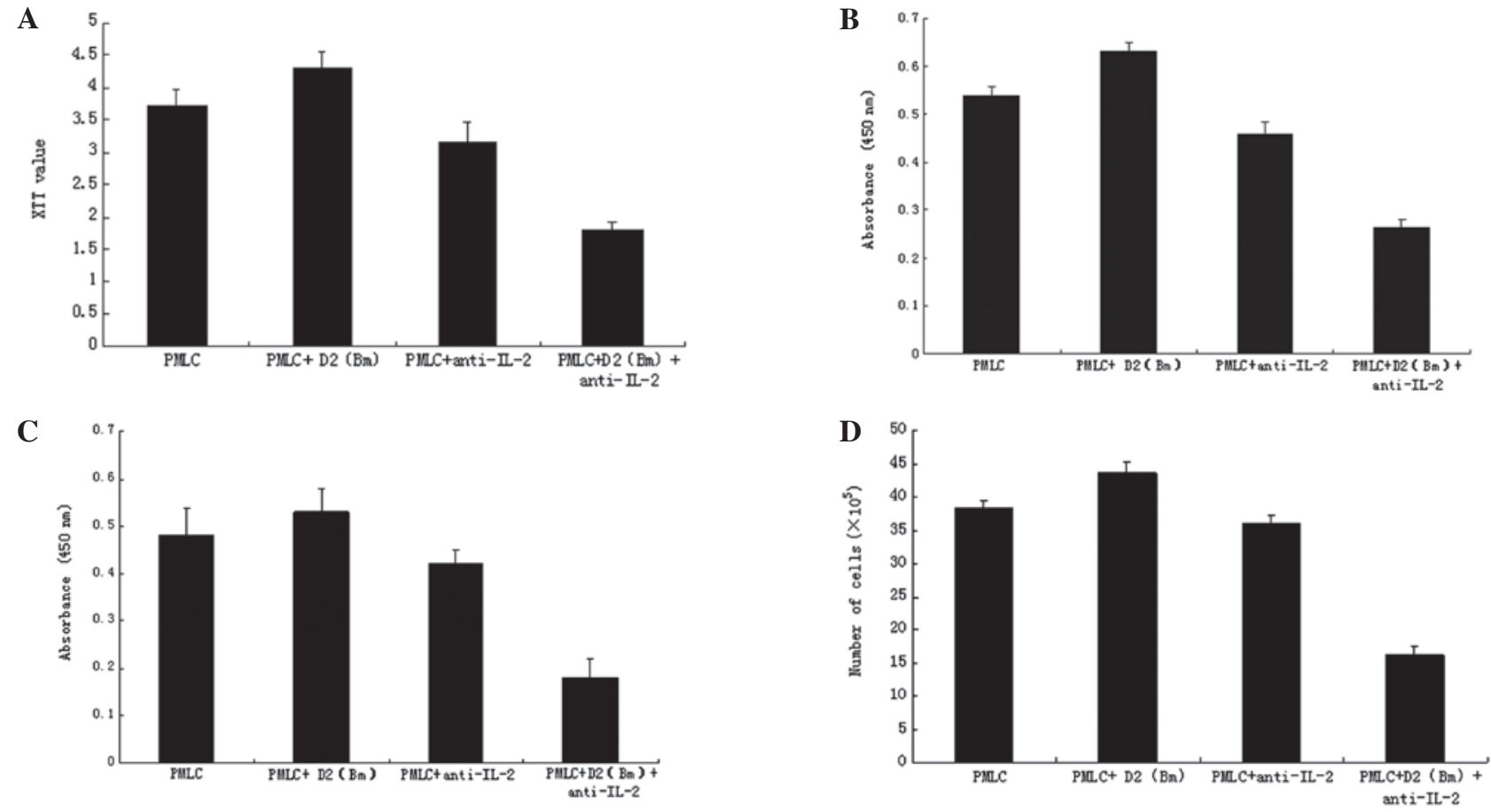

Figure 2. Anti-IL-2 neutralizing antibody inhibits lymphocyte growth and proliferation. (A) By XTT assays, the cell growth in the PMLC + D2 (Bm) + anti-IL-2 group was markedly lower than that in the PMLC and PMLC + D2 (Bm) group ( $\mathrm{P}<0.01)$, but not in the PMLC + anti-IL-2 group. (B) The lymphocyte DNA synthesis analyzed using BrdU showed that in the PMLC+D2 (Bm) + anti-IL-2 group, DNA change was markedly lower than in the PMLC and PMLC + D2 (Bm) groups ( $<<0.01)$, but not in the PMLC+anti-IL-2 group. (C) Cell viability was evaluated by CCK-8 assay and (D) lymphocyte proliferation was evaluated by cell count, the results of which were similar to XTT assays. The lymphocytes in the PMLC+anti-IL-2 group ( $<<0.01)$ were significantly inhibited but not in the PMLC+D2 (Bm) and PMLC+anti-IL-2 groups $(\mathrm{P}>0.05)$.

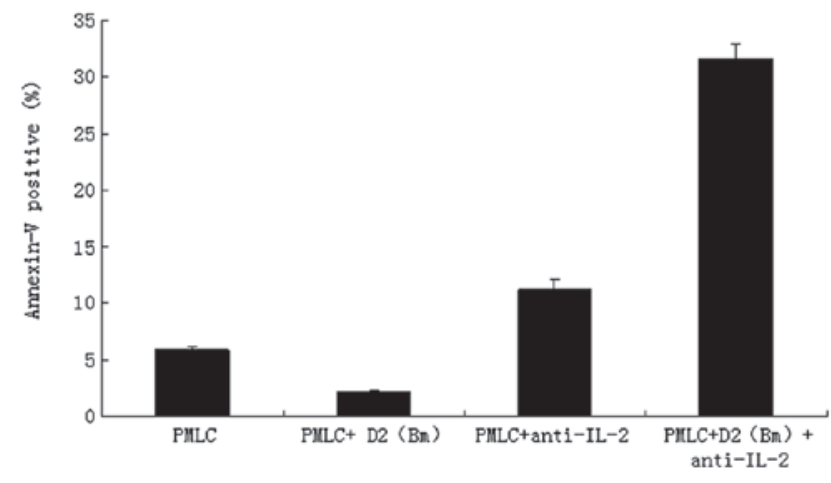

Figure 3. Anti-IL-2 neutralizing antibody promotes lymphocyte apoptosis. The effect of mixed lymphocyte culture and/or addition of IL-2 purified neutralizing monoclonal antibody on apoptosis distribution was determined by flow cytometry. The apoptosis lymphocytes in the PMLC + anti-IL-2 $(11.25 \pm 0.78 \%)$ and PMLC + D2 $(\mathrm{Bm})+$ anti-IL-2 groups $(31.65 \pm 1.33 \%)$ were markedly higher than in the PMLC $(5.95 \pm 0.24 \%)$ or PMLC + D2 $(\mathrm{Bm})$ groups $(2.15 \pm 0.04 \%)$ separately $(\mathrm{P}<0.01)$. The difference between the PMLC + anti-IL-2 and PMLC + D2 (Bm) + anti-IL-2 groups was also statistically significant $(\mathrm{P}<0.01)$. The results showed that mixed lymphocyte culture and/or addition of IL-2 purified neutralizing monoclonal antibody significantly induced lymphocyte apoptosis.

p53 and caspase-3 were involved in recipient lymphocyte apoptosis. In the PMLC + anti-IL-2 and PMLC + D2 $(\mathrm{Bm})+$ anti-IL-2 groups, the expression of p53 increased significantly, particularly in the latter. The evidence that lymphocyte apoptosis is induced by MLC and IL-2 purified neutralizing monoclonal antibody, particularly by the latter, and is associated with caspase- 3 activated cleavage products is shown in Fig. 4. Overall, these results indicate that MLC

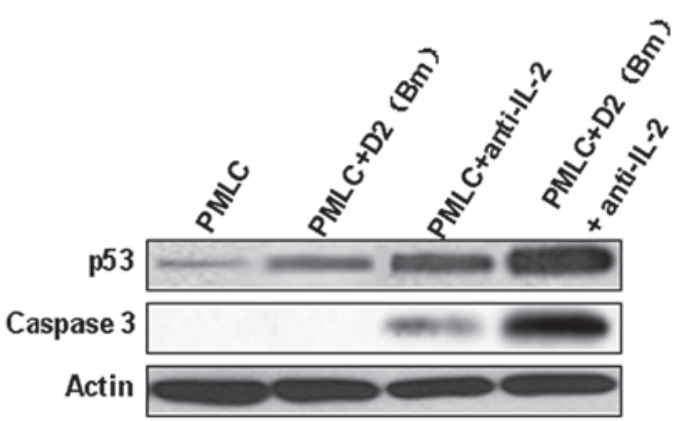

Figure 4. p53 and caspase- 3 are involved in lymphocytes apoptosis. In the PMLC + anti-IL-2 and PMLC + D2 (Bm) + anti-IL-2 groups, the expression of p53 increased significantly, particular in the latter. The lymphocyte apoptosis induced by mixed lymphocyte culture and addition of IL-2 purified neutralizing monoclonal antibody were identified to be associated with caspase- 3 activated cleavage products, particularly when anti-IL-2 was added. Overall, these results indicate that mixed lymphocyte culture and addition of IL-2 purified neutralizing monoclonal antibody promote apoptosis of mixed culture lymphocytes via pathways involving p 53 and caspase-3.

and IL-2 purified neutralizing monoclonal antibody promotes lymphocyte apoptosis via p53 and caspase-3 pathways (Fig. 4).

\section{Discussion}

Transplant rejection is a complex and redundant response to grafted organs. Its major targets are the major histocompatibility complex (MHC) antigens, which are designated as human leukocyte antigens (HLAs). T lymphocytes recognize 
foreign antigens only when the antigen or an immune peptide is associated with a self-HLA molecule on the surface of an accessory cell known as an antigen-presenting cell (APC). To resolve the immune rejection problem ahead of organ transplantation, we established a lymphocyte inhibition model by co-culturing mixed lymphocytes to induce recipient cell apoptosis and immune tolerance.

The main type of inducible cell death is apoptosis. Apoptosis is a type of programmed cell death (18) and is regulated by a series of signal cascades in an orderly manner. The caspase cascade response plays a vital role in the induction, transduction and amplification of intracellular apoptotic signals (19). Caspase-3 is a key factor in apoptosis execution, being responsible either partially or completely for the proteolytic cleavage of numerous key proteins (20).

In addition, p53 also plays a fundamental regulatory role in apoptosis, and induces apoptosis by caspase activation (21). The p53 protein, which is encoded by the TP53 gene, has a broad range of biological functions, including regulation of cell cycle, apoptosis, senescence, DNA metabolism, angiogenesis, cellular differentiation and the immune response. The key role of p53 is to induce apoptosis in response to cellular stresses such as DNA damage (22). Numerous studies have described the mechanism by which p53 induces apoptosis. As p53 functions mainly as a transcription factor, it is important to explore the genes regulated by $\mathrm{p} 53$ that contribute to the regulation of apoptosis. Apoptosis occurs through one of two major pathways, described as either the intrinsic mitochondrial or extrinsic death receptor pathway. In the mitochondrial pathway, death stimuli target mitochondria either directly or through transduction by proapoptotic members of the Bcl-2 family, including Bax and Bak. The mitochondria then release apoptogenic proteins, ultimately leading to caspase activation and apoptosis. In the death receptor pathway, following interactions with their cognate ligands, the receptors located at the cellular membrane recruit adaptor proteins such as initiator caspase-8, triggering the activation of caspases to orchestrate apoptosis. The crosstalk between the pathways is mediated via Bid, and possibly other factors that mediate cell death by modulation of the two pathways and perhaps other unrecognized pathways.

Immune tolerance is important in organ transplantation. Apoptosis of lymphocytes plays a critical role in central immune tolerance and peripheral immune tolerance, and is a type of programmed cell death. It is a well-known fact that the properly functioning immune system is dependent on programmed cell death at every developmental stage of the lymphocyte and for its activity. Activation-induced cell death (AICD) is the process by which cells undergo apoptosis in a controlled manner through the interaction of a death factor and its receptor $(23,24)$.

IL-2 plays an important and complex role in the immune system, serving as growth factor, differentiation factor and regulator of cell death. The endogenous expression of IL-2 is increased by the costimulation of previously activated T lymphocytes, which increases the expression of Bcl-2 and inhibits AICD of previously activated T lymphocytes (25). However, certain researchers observed the discrepant phenomenon that IL-2 leads to increased susceptibility to AICD (26-29).
In our research, by using MLC and IL-2 neutralizing monoclonal antibody, we successfully developed an in vitro lymphocyte inhibition model. We demonstrated that antigens and IL-2 neutralizing monoclonal antibody had different inhibitory effects on the lymphocyte activities in various mixed ways. The results showed that the lymphocyte activity was very high when adding antigens alone [in the PMLC + D2 (Bm) group], but was markedly lowered when adding antigens and IL-2 neutralizing monoclonal antibody together [in the PMLC + D2 $(\mathrm{Bm})+$ anti-IL-2 group]. Adding IL-2 neutralizing monoclonal antibody alone (in the PMLC + anti-IL-2 group) also inhibited lymphocyte activity, but the depression effect was weaker than adding antigens and IL-2 neutralizing monoclonal antibody together. By combining the TEM technique, Giemsa-Wright stain and flow cytometry, we found that the depressed lymphocyte activity was due to the enhancement of apoptosis. Similar to the results above, we observed various ratios of apoptotic cells in the different groups. With the exception of the control group, the ratio of apoptotic cells was highest in the PMLC + D2 $(\mathrm{Bm})+$ anti-IL-2 group and lowest in the PMLC + D2 $(\mathrm{Bm})$ group. The presence of IL-2 neutralizing monoclonal antibody was associated with more apoptotic activated lymphocytes in our experiment, which suggested that IL-2 may inhibit apoptosis of lymphocytes.

In our study, we first observed the different level of apoptosis of lymphocytes among different groups, and subsequently attempted to find the dominant mechanism related to apoptosis. As we know, induction of apoptosis is an essential function of p53 as a tumor suppressor $(22,30,31)$. The protein p53 can activate its downstream targets in a sequence-specific manner to induce apoptosis. The majority of tumor-derived p53 mutants are deficient in transcription activation as well as apoptosis induction. p53 can activate genes in the extrinsic and intrinsic pathways through transcription-dependent mechanisms or induce apoptosis through transcription-independent mechanisms. Results showed that the expression of p53 and cleaved caspase- 3 increased markedly in the PMLC + D2 $(\mathrm{Bm})+$ anti-IL-2 group, while there was an extremely low level of p53 and cleaved caspase-3 in the PMLC + D2 (Bm) group, which was in accordance with the ratio of apoptotic lymphocytes.

According to these results, we can summarize a novel mechanism of immune rejection: when the IL-2 from activated lymphocytes is blocked with neutralizing antibody, the activated lymphocytes undergo p53-induced apoptosis.

\section{Acknowledgements}

This study was supported by a grant from the National Natural Science Foundation of China (no. 30600524).

\section{References}

1. Helderman JH, Hernandez J, Sagalowsky A, et al: Confirmation of the utility of fine needle aspiration biopsy of the renal allograft. Kidney Int 34: 376-381, 1988.

2. Von Willebrand E and Hughes D: Fine-needle aspiration cytology of the transplanted kidney. In: Kidney Transplantation. Morris PJ (ed). 4th edition. WB Saunders, Philadelphia, PA, p301, 1994.

3. Suthanthiran M: Clinical application of molecular biology: a study of allograft rejection with polymerase chain reaction. Am J Med Sci 313: 264-267, 1997. 
4. Rimm IJ, Krenger W, Beland JL, et al: TCR-beta transgenic mice fail to mediate a GVHR due to defects of allorecognition and subsequent IL-2 generation. Bone Marrow Transplant 17: $835-842,1996$

5. Koreth J, Matsuoka K, Kim HT, et al: Interleukin-2 and regulatory T cells in graft-versus-host disease. N Engl J Med 365 2055-2066, 2011.

6. Zhang S, Dai H, Wan N, et al: Manipulating IL-2 availability amid presentation of donor MHC antigens suppresses murine alloimmune responses by inducing regulatory $\mathrm{T}$ cells. PLoS One 5: e8756, 2010

7. Webster KE, Walters S, Kohler RE, et al: In vivo expansion of $T$ reg cells with IL-2-mAb complexes: induction of resistance to EAE and long-term acceptance of islet allografts without immunosuppression. J Exp Med 206: 751-760, 2009.

8. Kang HG, Zhang D, Degauque N, et al: Effects of cyclosporine on transplant tolerance: the role of IL-2. Am J Transplant 7: 1907-1916, 2007

9. Sebille F, Brouard S, Petzold T, et al: Tolerance induction in rats, using a combination of anti-CD154 and donor splenocytes, given once on the day of transplantation. Transplantation 75: 169-172, 2003.

10. Subbotin V, Sun H, Aitouche A, et al: Abrogation of chronic rejection in a murine model of aortic allotransplantation by prior induction of donor-specific tolerance. Transplantation 64: 690-695, 1997.

11. Shen Z, Mohiuddin M, Goldstein C, et al: Durability of donorspecific and organ-specific heart transplant tolerance induced by intrathymic pretreatment with allogeneic spleen cells. J Thorac Cardiovasc Surg 111: 429-431, 1996.

12. Opelz G and Terasaki PI: Poor kidney-transplant survival in recipients with frozen-blood transfusions or no transfusions Lancet 2: 696-698, 1974.

13. Kroemer G, Galluzzi L and Brenner C: Mitochondrial membrane permeabilization in cell death. Physiol Rev 87: 99-163, 2007.

14. Sheehy MJ, Sondel PM, Bach ML, et al: HL-A LD (lymphocyte defined) typing: a rapid assay with primed lymphocytes. Science 188: 1308-1310, 1975 .

15. Amicarelli F, Bucciarelli T, Poma A, et al: Adaptive response of human melanoma cells to methylglyoxal injury. Carcinogenesis 19: 519-523, 1998

16. Ford J, Jiang M and Milner J: Cancer-specific functions of SIRT1 enable human epithelial cancer cell growth and survival. Cancer Res 65: 10457-10463, 2005

17. Hamamoto R, Furukawa Y, Morita M, et al: SMYD3 encodes a histone methyltransferase involved in the proliferation of cancer cells. Nat Cell Biol 6: 731-740, 2004.
18. Kerr JF, Wyllie AH and Currie AR: Apoptosis: a basic biological phenomenon with wide-ranging implications in tissue kinetics. Br J Cancer 26: 239-257, 1972

19. Thornberry NA and Lazebnik Y: Caspases: enemies within. Science 281: 1312-1316, 1998.

20. Krajewska M, Wang HG, Krajewski S, et al: Immunohistochemical analysis of in vivo patterns of expression of CPP32 (Caspase-3), a cell death protease. Cancer Res 57: 1605-1613, 1997.

21. Amaral JD, Castro RE, Sola S, et al: p53 is a key molecular target of ursodeoxycholic acid in regulating apoptosis. J Biol Chem 282: 34250-34259, 2007.

22. Amaral JD, Xavier JM, Steer CJ, et al: The role of p53 in apoptosis. Discov Med 9: 145-152, 2010.

23. Shi YF, Sahai BM and Green DR: Cyclosporin A inhibits activation-induced cell death in T-cell hybridomas and thymocytes. Nature 339: 625-626, 1989.

24. Lieberman AC, Refojo D, Antunica-Noguerol M, et al: Underlying mechanisms of cAMP- and glucocorticoid-mediated inhibition of FasL expression in activation-induced cell death. Mol Immunol 50: 220-235, 2012.

25. Pender MP: Activation-induced apoptosis of autoreactive and alloreactive $\mathrm{T}$ lymphocytes in the target organ as a major mechanism of tolerance. Immunol. Cell Biol 77: 216-223, 1999.

26. Lawetzky A, Kubbies M and Hünig T: Rat 'first-wave' mature thymocytes: cycling lymphoblasts that are sensitive to activationinduced cell death but rescued by interleukin 2. Eur J Immunol 21: 2599-2604, 1991.

27. Miller AT and Berg LJ: Defective Fas ligand expression and activation-induced cell death in the absence of IL-2-inducible T cell kinase. J Immunol 168: 2163-2172, 2002.

28. Maher SG, Condron CE, Bouchier-Hayes DJ and Toomey DM: Taurine attenuates CD3/interleukin-2-induced T cell apoptosis in an in vitro model of activation-induced cell death (AICD). Clin Exp Immunol 139: 279-286, 2005.

29. Curtale G, Citarella F, Carissimi C, et al: An emerging player in the adaptive immune response: microRNA-146a is a modulator of IL-2 expression and activation-induced cell death in T lymphocytes. Blood 115: 265-273, 2010.

30. Liebermann DA, Hoffman B and Vesely D: p52 induced growth arrest versus apoptosis and its modulation by survival cytokines. Cell Cycle 6: 166-170, 2007.

31. Olivier M, Petitjean A, Marcel V, et al: Recent advances in p53 research: an interdisciplinary perspective. Cancer Gene Ther 16: $1-12,2009$. 